\title{
Sensitivity of IceCube Cosmic-Ray measurements to the hadronic interaction models
}

\author{
The IceCube Collaboration ${ }^{\dagger}$ \\ $\dagger$ http://icecube.wisc.edu/collaboration/authors/icrc17_icecube \\ E-mail: sam.deridder@ugent.be
}

\begin{abstract}
The IceCube Neutrino Observatory measures cosmic-ray air showers with both its surface array IceTop and its 1.5-2.5 km deep in-ice array. IceTop measures the charge deposited by electromagnetic particles and low-energy muons. The highly abundant electromagnetic particles mainly determine the cosmic ray primary energy, while the low-energy muons, visible at the edge of the shower, add a sensitivity to the primary composition. The high-energy ( $>300 \mathrm{GeV})$ muon bundle is studied through its energy loss profile in the in-ice detector. These muons provide information about the early interactions of the cosmic ray in the atmosphere and thus to the mass of the primary particle. In this work we combine all three pieces of information. Since the yield of low- and high-energy muons differs significantly among existing hadronic interaction models, this provides a unique sensitivity to model dependent variations in cosmic-ray air shower studies.

Corresponding authors: Sam De Ridder ${ }^{1}$, Emily Dvorak ${ }^{2}$, Tom Gaisser ${ }^{* 3}$

${ }^{1}$ Dept. of Physics and Astronomy, University of Gent, B-9000 Gent, Belgium

2 Physics Department, South Dakota School of Mines and Technology, Rapid City, SD 57701, USA

${ }^{3}$ Bartol Research Institute and Dept. of Physics and Astronomy, University of Delaware, Newark, DE 19716, USA
\end{abstract}

35th International Cosmic Ray Conference - ICRC2017

10-20 July, 2017

Bexco, Busan, Korea

${ }^{*}$ Speaker. 


\section{Introduction}

Indirect measurements of cosmic rays (CR) with energies $>1 \mathrm{PeV}$ require a good understanding of the propagation of extensive air showers (EAS) in the atmosphere. This is a complex process with millions of particle interactions, so one needs to rely on simulations to relate experimental observables to the properties of the primary cosmic ray. Cosmic-ray interactions with nuclei in the atmosphere however occur around and above the highest energies that can be reached in presentday accelerators. The Large Hadron Collider (LHC) operates at a center-of-mass energy of $14 \mathrm{TeV}$, which corresponds to a lab-frame energy of roughly $100 \mathrm{PeV}$. On top of this, the very forward region with small transverse momenta of the secondary products of a collision is not well covered in the CMS and ATLAS detectors, while it is the most important region in cosmic ray physics. Air shower simulations thus need to rely on extrapolations of LHC data and fixed-target experiments at lower energies. This of course induces uncertainties in the interaction cross-sections, secondary particle type, multiplicity, particle momenta, etc. The assumptions and approximations used will influence the measurements of EAS properties by experiments.

Experiments reconstructing the cosmic-ray energy and mass use the variations between models used in simulations as a systematic uncertainty on their measurement. However, composition results between various experiments diverge significantly even with these systematic errors included. In particular, IceCube observes a rather heavy composition above $100 \mathrm{PeV}$ to $1 \mathrm{EeV}$ ([1]), while other experiments obtain a more light composition. In view of the uncertainties within the hadronic interactions, it is important to consider that these experiments measure different parts of the EAS. IceCube used the number of high-energy $(>300 \mathrm{GeV})$ muons as composition probe, while other experiments relate the muon content on the surface or the depth of the shower maximum to the primary mass.

In this work we study the extent to which varying results could arise from the fact that different experiments use different EAS observables. By comparing multiple observables within one single measurement, the internal (in)-consistencies concerning composition results within models can be examined. Here we will specifically focus on the comparison between a composition reconstruction using the surface detector array and in-ice measurements from the IceCube Neutrino Observatory (described in Sections 2 and 3). In particular, a composition estimation using the slope of the lateral distribution function at the surface is compared to the high-energy (HE) muon bundle information. The results obtained from these variables highly depend on the muon spectra produced in simulations, which differ among the hadronic interaction models (Section 4). The corresponding implications for the observables under study are shown in Section 5. In Section 6 data is added to compare whether the observables predict a similar composition behaviour under a given hadronic model.

\section{Cosmic ray measurements with the IceCube Neutrino Observatory}

The IceCube Neutrino Observatory [2] consists of a deep in-ice component "IceCube" (IC), together with a surface array called "IceTop" (IT) [3] (Figure 1). It is located at the geographic South Pole, at about $1 \mathrm{~km}$ from the Amundsen-Scott South Pole station. The in-ice component consists of 86 strings of which 78 have a standard inter-string spacing of $125 \mathrm{~m}$. At depths between 


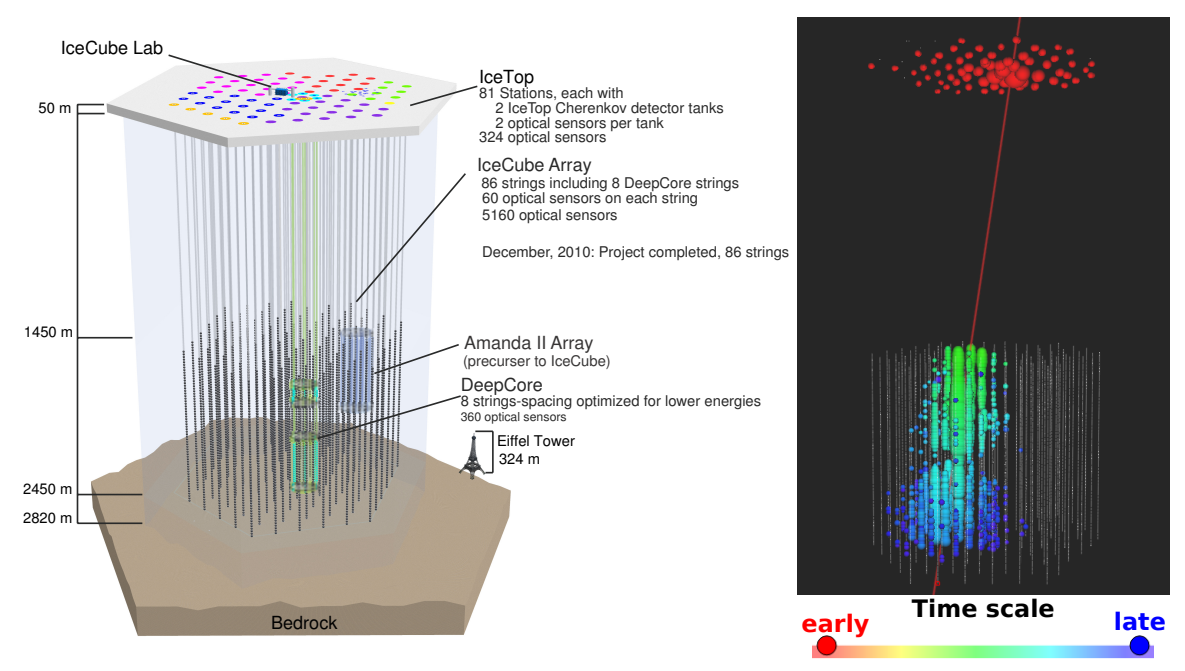

Figure 1: Schematic view of the IceCube Neutrino Observatory and its components, together with an illustration of the signal deposition by a real air shower event in both IceTop and IceCube.

1450 and 2450 meter below the Antarctic surface 60 digital optical modules (DOMs) are deployed on each string. In total IceCube covers a volume of $1 \mathrm{~km}^{3}$, which makes it very well suited for its primary goal: neutrino astronomy. At the surface above the in-ice detector 81 IceTop stations are deployed. Most stations are located close to the top of an IceCube string and hence they are spread over $1 \mathrm{~km}^{2}$ with an inter-station distance of $\sim 125 \mathrm{~m}$. Each IceTop station consists of two ice Cherenkov tanks with a $10 \mathrm{~m}$ separation. Operating these tanks in coincidence reduces a significant fraction of the background noise. In this work we only use the tank signals if such a coincident requirement between both tanks is fullfilled (called "hard local coincidence" or HLC hits). Calibration of the tanks is performed using the equivalent signal produced by a vertical muon going through the tank (VEM). The tanks themselves contain two DOMs which operate at different gains to increase the dynamic range for the detection of EAS $(\mathrm{O}(0.1)-\mathrm{O}(1000)$ VEM). The right plot of Figure 1 shows an example of a large EAS detection using IT and IC. Since IceTop is located at a height of $2835 \mathrm{~m}$ above sea level, its average atmospheric depth of $692 \mathrm{~g} / \mathrm{cm}^{2}$ is rather close to the depth of shower maximum for showers between $1 \mathrm{PeV}$ and $1 \mathrm{EeV}$. This results in a large amount of EM particles, and accordingly, smaller statistical fluctuations. The abundant electromagnetic (EM) component and the low-energy muons first create a footprint on the IceTop array. The bundle of HE muons $(>300 \mathrm{GeV})$, created in the first EAS interactions, travels 1.5 $\mathrm{km}$ through the ice and deposits energy along its track. The IceCube Neutrino Observatory is operational in its full configuration since May 2011. In this work only one month of data will be used to avoid influence of seasonal variations, being April 2013. This is a month from the second full year of data-taking for IceCube (May 2012 - May 2013), which corresponds to the data-taking year in the used simulations. The energy threshold at which cosmic ray detection becomes fully efficient for this data-taking year is $\log _{10}(\mathrm{E} / \mathrm{GeV})=6.4$. This threshold is slowly increasing with energy due to the accumulation of snow on top of the tanks. The effective detector area becomes too small for a detection of a significant rate of cosmic rays with energies $>1 \mathrm{EeV}$. However, this work only focuses on the energy region up to $\sim 100 \mathrm{PeV}$. 

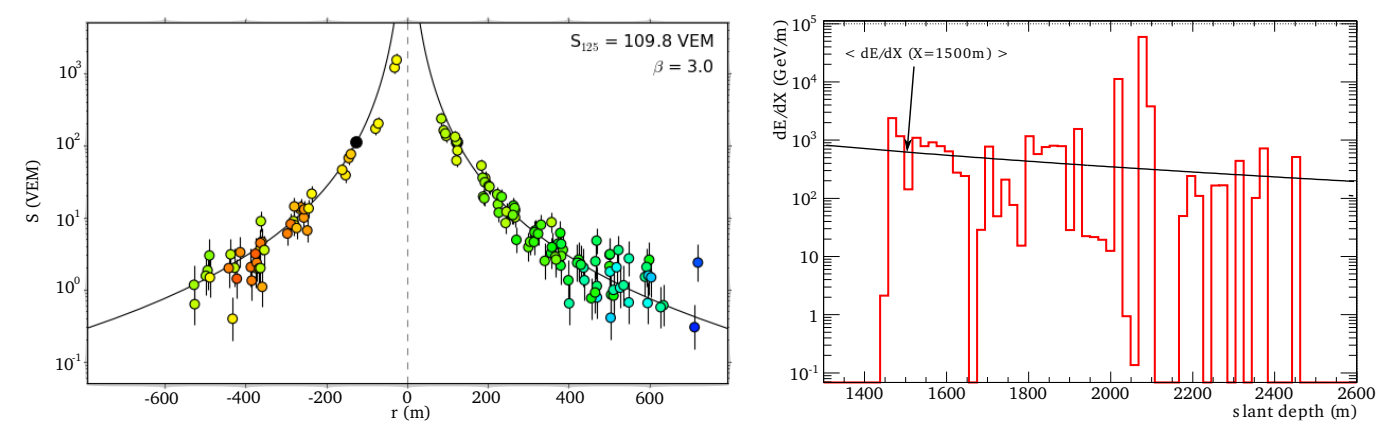

Figure 2: Left: Fit to the lateral charge distribution (LDF) in IceTop. Colours again indicate the time of the tank signal. Right: Fit to the energy loss profile along the track of the muon bundle in the ice.

\section{EAS reconstruction}

The air shower reconstruction performed here is very similar to the standard method used in IceTop analyses [3]. If 5 stations with HLC pulses survive after noise removal algorithms, a loglikelihood minimization technique tries to find the core position, direction (with zenith angle $\theta$ ) and several parameters describing the shower. A lateral distribution function (LDF) is fitted to the charge as function of distance to the shower axis (R) (Figure 2), in combination with a curvature function describing the shower front time. The LDF used to fit the measured charges $\left(S_{\text {meas }}\right)$ is a double logarithmic parabola:

$$
S_{\text {meas }}(R)[\mathrm{VEM}]=S_{125} \cdot\left(\frac{R}{125 m}\right)^{-\beta-0.303 \log _{10}\left(\frac{R}{125 m}\right)} \cdot \exp \left(\frac{-d_{\text {snow }} \sec \theta}{\lambda}\right)
$$

The two free parameters of this LDF are $\mathrm{S}_{125}$ and $\beta$, which are respectively the signal size and slope of the LDF at $125 \mathrm{~m}$. The last part of Eq. 3.1 handles the attenuation of the signal due to the snow (with height $d_{\text {snow }}$ ) on top of each tank. The attenuation length $\lambda$ is an effective attenuation length correcting both the muonic signal and EM signal, and its value is found to be $2.25 \pm 0.2 \mathrm{~m}$ for this data-taking year. Next to the requirement of having at least 5 HLC stations after event cleaning, additional cuts are applied in order to ensure a set of properly described events on the surface. These cuts mainly concern the reconstruction quality and containment within the IceTop array. When the IceTop related cuts are fulfilled, the EAS trajectory reconstructed on the surface is used as muon bundle trajectory in the ice. Only hits that are related to the muon bundle track in time and space are selected to remove noise and random coincidences. If more than 8 in-ice HLC hits pass this procedure, a reconstruction of the energy loss profile along the track is performed, using the method described in [4] (Figure 2). A fit to this energy loss profile is performed in order to obtain a reliable energy loss reconstruction. The reconstructed energy loss at a slant depth of $1500 \mathrm{~m}$ in the ice $\left(d E / d X_{1500}\right)$ is a good measure for the number of HE muons in the air shower. More cuts are applied to the in-ice energy loss reconstruction to ensure that the track traverses a significant part of the detector and that the reconstructed energy loss profile describes the measured light yield well. Furthermore, the fit to the energy loss profile must have converged. 

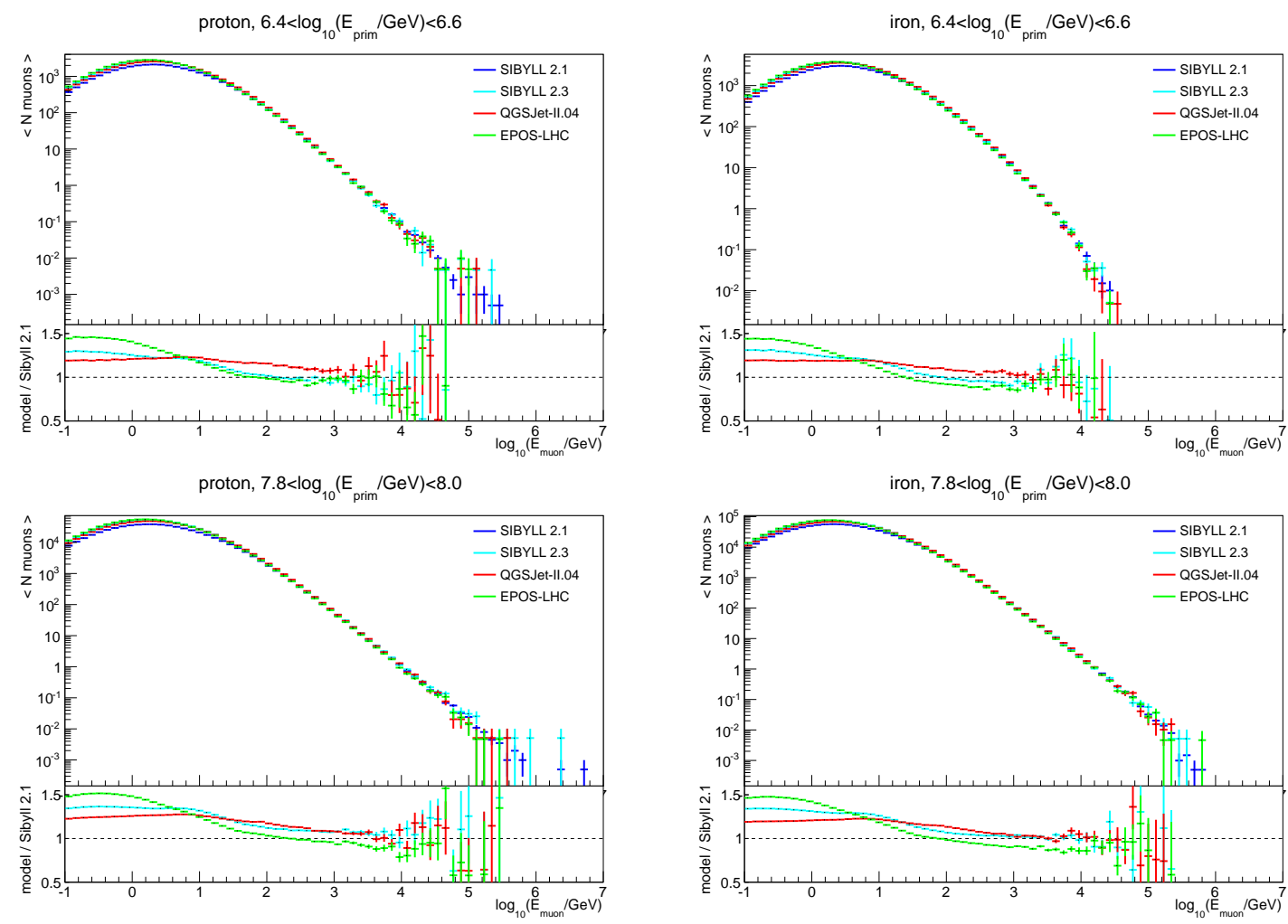

Figure 3: Spectrum of surface muons at South Pole altitude $\left(692 \mathrm{~g} / \mathrm{cm}^{2}\right.$, April 2011 South Pole atmosphere) for proton and iron showers with zenith angles $<40^{\circ}$ in two energy ranges. The spectra obtained with various hadronic models are shown, together with the ratio with respect to SIBYLL 2.1.

\section{Hadronic interaction models}

The simulation of the EAS development in the atmosphere is performed using the standard CORSIKA simulation package [5]. The atmospheric profile used in these simulations is the average South Pole atmosphere of April 2011, which represents the mean profile of an entire data-taking year. The propagation of the EM component is treated using EGS4, while hadronic interactions with energies $<80 \mathrm{GeV}$ are modelled using FLUKA [6]. At higher energies, the hadronic interactions are modelled with SIBYLL 2.1 [7] in the standard dataset (with the largest statistics). The other hadronic models under study are the post-LHC models: SIBYLL 2.3 [8], QGSJet-II.04 [9] and EPOS-LHC [10]. These models are mainly tuned on the latest LHC data, but the most observable change for EAS measurements with IT-IC is their enhancement of muons. Figure 3 shows the resulting muon spectra that are obtained after simulations down to an atmospheric depth corresponding to the IceTop altitude $\left(692 \mathrm{~g} / \mathrm{cm}^{2}\right)$. The spectra are plotted for proton and iron primaries in two energy bins, and the various colors represent the different hadronic models. The bottom of each plot shows the muon number increase relative to SIBYLL 2.1. The curves show the average over many showers and error bars describe the error on the average. The number of low-energy muons increases for all new hadronic models, for both primaries and energy bins. The largest increase in LE (surface) muon number is observed for EPOS-LHC ( $>40 \%)$. This number increases 
by $\sim 30 \%$ and $\sim 20 \%$ for respectively SIB YLL 2.3 and QGSJet-II.04. The change in the number of $\mathrm{HE}$ muons $(>300 \mathrm{GeV})$ is smaller. On average there seems to be a decrease of $5-10 \%$ for simulations using EPOS-LHC, while both QGSJet-II.04 and SIBYL 2.3 only show a very small, nearly negligible, increase in HE muon number relative to SIBYLL 2.1.

\section{Parameter description and model sensitivity}

The shower size parameter $S_{125}$ scales with the number of (EM) particles in the shower and thus the energy of the primary particle. In this work, $\log _{10}\left(S_{125} / \mathrm{VEM}\right)$ ranges between 0.4 and 2 , which roughly translates to a primary energy range of $6.4<\log _{10}\left(E_{\text {prim }} / \mathrm{GeV}\right)<8.125 \mathrm{~m}$ as reference distance was chosen in order to minimize the dependence of this parameter to the primary composition. $\beta$, the slope of the LDF at this same radius, on the other hand does show sensitivity to the mass of the primary particle. EAS induced by heavier nuclei generally interact higher in the atmosphere and develop faster, or are older in terms of shower age, therefore the shower will be more spread out and the slope of the LDF is smaller. Additionally EAS initiated by heavier CRs contain more muons (both LE and HE) compared to lighter nuclei, which also results in a flatter LDF. Both these effects thus result in a composition sensitivity of $\beta$, where $\beta$ is smaller for higher masses. The number of HE muons contained in the muon bundle is parametrized using the $\log _{10}\left(d E / d X_{1500}\right)$ observable. Since the number of HE muons in a shower increases with primary composition, also this variable shows a composition sensitivity. Both variables change linearly with $\log (\mathrm{A})$, where $\mathrm{A}$ is the mass number of the primary particle. The variables are clearly complementary in a reconstruction of the primary cosmic ray composition, since they are sensitive to various parts of the EAS. The distributions of both variables $\beta$ and $\log _{10}\left(d E / d X_{1500}\right)$ within a certain $\log _{10}\left(S_{125}\right)$ bin are fitted with a gaussian distribution. For both variables, the spread decreases with energy. The mean values of these gaussian fits are shown in Figure 4 as function of $\log _{10}\left(S_{125}\right)$ for both $\beta$ and $\log _{10}\left(d E / d X_{1500}\right)$. Again, the error bars show the error on the mean. The solid (dashed) lines show proton (iron) and the separation between the two indicates a clear composition sensitivity of both variables.

The results obtained with the various hadronic models are shown as the various colours, and the difference with SIBYLL 2.1 is plotted on the bottom. The difference between QGSJet-II.04, SIBYLL 2.3 and SIBYLL 2.1 concerning HE muons is small (Figure 3), which results in a negligible change in the measured energy loss in the ice. For EPOS-LHC on the other hand a significant reduction can be observed. The decrease in $\beta$ with regard to SIBYLL 2.1 is observable for all post-LHC hadronic interaction models. Again EPOS-LHC shows the largest shift. This decrease is mostly correlated with the increased number of surface muons discussed in Section 4.

The observed light yield, and thus the reconstructed energy loss, highly depends on the properties of the ice. The uncertainty on those properties will result in a systematic error on $\log _{10}\left(d E / d X_{1500}\right)$. In this work the systematic errors caused by scattering and absorption uncertainties in the main ice, the scattering length of the refrozen ice surrounding the strings, and the efficiency of the DOMs are combined into a general light yield error of $-12.5 \%$ and $+9.6 \%$. The uncertainty of the snow attenuation length $(0.2 \mathrm{~m})$ is combined with the systematic error due to the uncertainty of the calibration of the tanks $( \pm 3 \%)$. Both of these mainly influence the signal size $S_{125}$, while the composition sensitive variables are almost unaffected. 

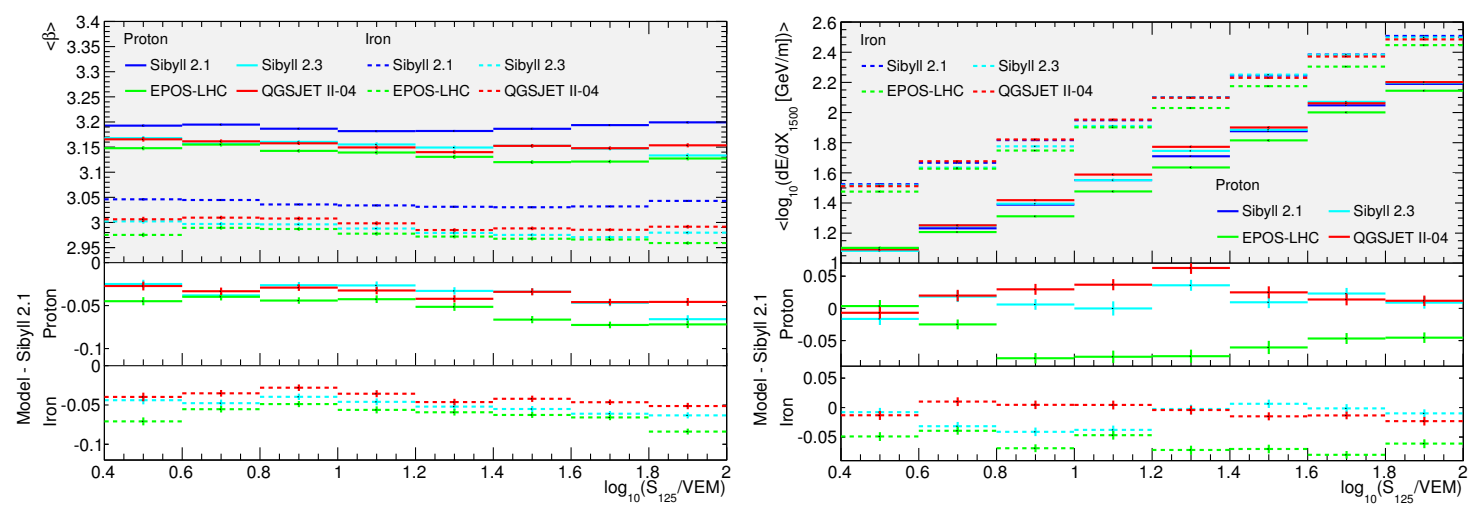

Figure 4: Evolution of the average $\beta$ and $d E / d X_{1500}$ as function of $S_{125}$ for proton and iron simulations with the various hadronic interaction models. On the bottom the shift with respect to SIBYLL 2.1 is shown.

\section{Results and discussion}

When the simulations describe the data well, measured data curves should be somewhere in between simulated proton and iron curves, or at least very close to them. To compare the data results of the two variables $\left(\beta\right.$ and $\log _{10}\left(d E / d X_{1500}\right)$ ), we plot the relative position of data within the proton-iron space for each variable. When data is similar to proton (iron) for a certain variable, it has a value close to 0 (1). If a consistent composition is measured between the two variables, the two curves should overlap. The resulting comparison of the curves is shown Figure 5 for each of the hadronic interaction models. The systematic error bars correspond to the ones described in the previous section. The light yield uncertainty in the ice dominates our systematics.

A clear behaviour that can be seen in all of these plots is that the curves for both variables rise with $S_{125}$ and thus with energy, indicating an increasingly heavy composition. In this energy range (from roughly $2.5 \mathrm{PeV}$ to $100 \mathrm{PeV}$ ) this has been observed by all experiments. According to which hadronic interaction model is used, these plots show a varying agreement between the two observables. For both SIBYLL 2.3 and QGSJet-II.04, a consistent interpretation is obtained between the two parameters. In this case a composition measurement using an observable sensitive to shower age and the muonic component on the surface agrees with a measurement of the in-ice energy loss caused by the HE muons, which is of great importance. Results obtained with simulations using SIBYLL 2.1 and EPOS-LHC on the other hand show some discrepancy concerning these measurements. Namely the lower number of LE muons on the surface in SIBYLL 2.1 causes the measured slope of the LDF to give a heavier composition than the signal in deep ice. In the case of EPOS-LHC, the decreased number of HE muons combined with the $50 \%$ increase in the LE muon number leads to the opposite inconsistency. From this it can be concluded that it is of crucial importance to understand and take into account the variations within certain models and in between models, especially when cosmic-ray composition results are compared where different properties of the extensive air showers are used to reconstruct the primary cosmic-ray mass.

More work is in progress to extend the energy range in this work to higher energies. In the full analysis, more data and simulations will be used. In addition a direct measurement of the surface muons [11] will be added as separate observable. 

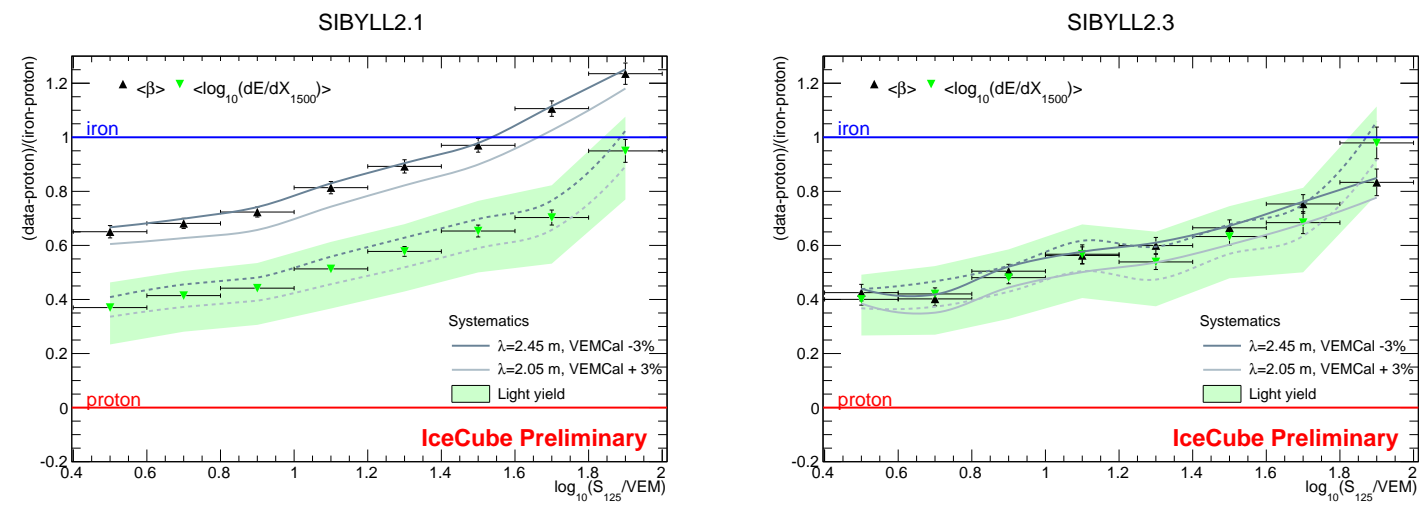

QGSJet-II.04

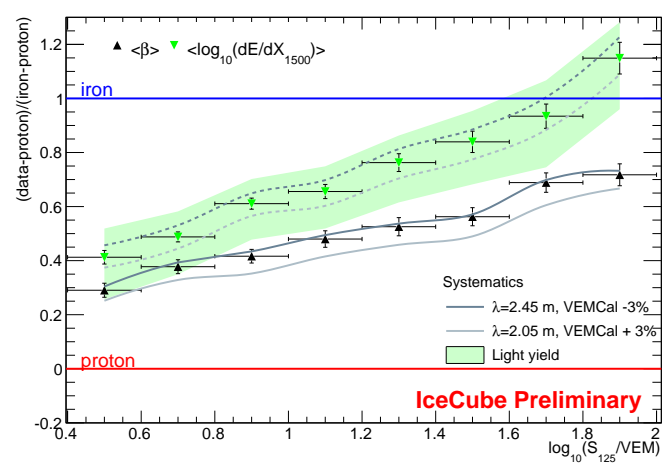

Figure 5: Distribution of the average of the $\beta$ and $\log _{10}\left(d E / d X_{1500}\right)$ measurements as function of $S_{125}$ in the proton-iron space. Each figure shows the results obtained with a certain hadronic model. The green band shows the in-ice light yield systematic, while the solid and dotted grey curves indicate uncertainties mainly in the energy scale from IceTop reconstructions.

\section{References}

[1] IceCube Collaboration, K. Rawlins, J. Phys. Conf. Ser. 718 (2016) 052033.

[2] IceCube Collaboration, A. Achterberg et al., Astropart. Phys. 26 (2006) 155-173.

[3] IceCube Collaboration, R. Abbasi et al., Nucl. Instrum. Meth. A700 (2013) 188-220.

[4] IceCube Collaboration, M. G. Aartsen et al., JINST 9 (2014) P03009.

[5] D. Heck et al., CORSIKA: A monte carlo code to simulate extensive air showers, tech. rep., 1998.

[6] A. Ferrari, P. R. Sala, A. Fasso“, and J. Ranft, CERN-2005-10 (2005). INFN/TC_05/11, SLAC-R-773.

[7] E.-J. Ahn, R. Engel, T. K. Gaisser, P. Lipari, and T. Stanev, Phys. Rev. D 80 (Nov, 2009) 094003.

[8] F. Riehn, R. Engel, A. Fedynitch, T. K. Gaisser, and T. Stanev, PoS ( ICRC2 015 ) 558 (2016).

[9] S. Ostapchenko, Phys. Rev. $\mathbf{D 8 3}$ (2011) 014018.

[10] T. Pierog, I. Karpenko, J. M. Katzy, E. Yatsenko, and K. Werner, Phys. Rev. C92 (2015) 034906.

[11] IceCube Collaboration, H. P. Dembinski and J. Gonzalez, Po S ( ICRC2015) 267 (2016). 